\title{
Electrophoretic Mobility of Latex Spheres in the Presence of Divalent Ions: Experiments and Modeling
}

\author{
Motoyoshi KoBAYASHI
}

Faculty of Agriculture, Iwate University, Ueda 3-18-8, Morioka, Iwate 020-8550, Japan

E-mail: mkoba@iwate-u.ac.jp

Fax \&Phone: 81-19-621-6190

\begin{abstract}
Electrophoretic mobilitiies (EPM) of negatively charged latex spheres were measured as a function of salt type and salt concentration. The measured values of EPM were analyzed using a standard electrokinetic model that includes double layer relaxation and the Poisson-Boltzmann model of diffuse double layer. Calculated values of EPM were in good agreement with experimental data taken in simple 1:1 $(\mathrm{KCl})$ and 1:2 $\left(\mathrm{Na}_{2} \mathrm{SO}_{4}\right)$ electrolyte solutions without using any fit parameters. For 2:1 electrolytes $\left(\mathrm{CaCl}_{2}\right.$ and $\left.\mathrm{MgCl}_{2}\right)$, however, the magnitude of EPM calculated by the model was higher than the measured values of EPM at higher electrolyte concentrations. The difference between measured and calculated EPM was reduced by assuming the distance of slipping plane $\mathrm{x}_{\mathrm{s}}=0.25 \mathrm{~nm}$ or by assuming the decrease of the magnitude of surface charge density from $-0.07 \mathrm{C} / \mathrm{m}^{2}$ to $-0.025 \mathrm{C} / \mathrm{m}^{2}$. These are probably due to the accumulation of divalent counter ions in the vicinity to particle's surface.
\end{abstract}

Keywords: Electrophoresis, Surface potential, Surface charge density, Latex, Relaxation 


\section{Introduction}

Various important properties of colloidal dispersion, such as stability, rheology, and adsorption etc, can be related to the charging behavior of colloidal particles [1-3]. Electrophoresis is a widely used technique to investigate charging properties of colloidal particles. In the technique, we measure the moving velocity of charged particles induced by an applied electric field, and obtain electrophoretic mobility (EPM), which is defined by the ratio of the migration velocity to the applied field.

The EPM reflects the sign and the magnitude of surface potential of colloidal particles. Nevertheless, it is not straightforward to interpret quantitatively surface potentials from EPM; theories are needed to convert EPM to electrokinetic or zeta potential $\zeta$. In the earliest studies, Smoluchowski [4], Henry [5], and Huckel [6] derived approximate expressions for the relationship $\zeta$ and EPM. Their theories are, however, limited to low surface potentials, and neglects the relaxation (polarization) effect of electric double layer (EDL). More elaborated theories were developed by Booth [7], Overbeek [8], Wiersema et al. [9] and O'Brien and White (OW) [10] to take into account the effect of relaxation of EDL surrounding a charged particle. While the OW theory requires numerical computation to be solved, some analytical approximate expressions are also available [11-14]. The theories predict the maximum of the magnitude of EPM in the relationship EPM versus $\zeta$, because the EDL polarization reduces the migration velocity of a highly charged particle.

Many experimental studies of EPM have been carried out using latex particles, which are considered as model colloidal particles, because the particles are spherical and monodisperse. Electrokinetic behavior of latex spheres, however, is often referred to as atypical or anomalous, that is, the magnitude of EPM has shown a maximum when plotted against electrolyte concentration [1520]. To explain this atypical mobility, several mechanisms such as specific co-ion adsorption, hairy layer and ion correlations, which are not included in the standard Poisson-Boltzmann (PB) and electrokinetic OW theories, have been proposed [1517, 21-23].

Antonietti and Vorwerg [17] measured the EPM of latex sphere, bearing a constant surface charge density, in $\mathrm{KCl}$ and $\mathrm{MgSO}_{4}$ solutions, and observed the 
maximum in the relationship between absolute values of EPM and electrolyte concentrations. In addition, they found that the maximum can be described by a simple theoretical calculation with the approximated version of OW theory, to calculate EPM from the surface potential, and the Gouy-Chapman (GC) theory derived from the PB equation, to convert charge density into surface potential. A similar conclusion has been made by Borkovec et al [18], who analyzed their mobility data of amidine latex in $\mathrm{KCl}$ solution by using the $\mathrm{GC}$ and the $\mathrm{OW}$ theories. While they used the standard (PB and OW) theory and did not take into account discrete natures of ions, theoretical calculations reasonably agreed well with experimental EPM values for larger particles. The PB and OW approach was also successful in predicting EPM behaviors of other particles in 1:1 electrolyte solutions [24, 25]. It is clear that the maximum is not atypical but results from a consequence of classical EDL and standard electrokinetic theories.

Recently, attention has been paid to the effect of multivalent counter-ions on EPM of colloidal particles. Quesada-Perez et al [21] analyzed EPM data of latex particles carrying a constant charge in $\mathrm{Mg}\left(\mathrm{NO}_{3}\right)_{2}, \mathrm{Ca}\left(\mathrm{NO}_{3}\right)_{2}$ and $\mathrm{La}\left(\mathrm{NO}_{3}\right)_{3}$ solutions. They have suggested that ion correlations must be included in theoretical calculations to obtain the quantitative agreement between theory and experiment in the presence of multivalent counter ions. By contrast, Takamura and Chow have shown the OW and PB approach reasonably describes the EPM of particles in emulsions, not in suspension, even in the presence of divalent counterions such as $\mathrm{Ca}^{2+}$ and $\mathrm{Mg}^{2+}$ [26]. However, they did not obtain good agreement between theory and experiment for the EPM of latex particles in the presence of divalent ions, and mainly focused on the shortcoming of the PB and OW theories at lower salt concentration [27]. Antonietti and Vorwerg [19] measured mobility only in symmetrical electrolyte solutions and did not find the quantitative agreement between theory and experiment, while they showed that the maximum of the magnitude of EPM results from the standard theories. At present, detailed discussion on the applicability of the standard PB and OW theories on the prediction of EPM of latex particles, especially in a solution of asymmetric electrolytes, is still lacking

In the present study, the EPM of sulfate latex spheres was measured in $\mathrm{KCl}, \mathrm{Na}_{2} \mathrm{SO}_{4}, \mathrm{CaCl}_{2}$, and $\mathrm{MgCl}_{2}$ solutions. Measured data were compared with 
the theoretical evaluations using the standard electrokinetic and PB theories to elucidate the validity of the theories.

\section{Experimental}

Commercially available surfactant free polystyrene sulfate latex spheres (1-2800, Interfacial Dynamics Corporation) were used as colloidal particles. The sulfate groups on the paricle surface are considered to be strong acid and thus to carry a constant negative charge irrespective of $\mathrm{pH}$ and ionic strength. The manufacturer reports that the diameter $2 a$, the density, and the surface charge density $\sigma$ of the spheres were $2.8 \pm 0.14 \mu \mathrm{m}, 1.055 \mathrm{~g} / \mathrm{cm}^{3}$, and $-0.07 \mathrm{C} / \mathrm{m}^{2}$, respectively. Four salt $\left(\mathrm{KCl}, \mathrm{Na}_{2} \mathrm{SO}_{4}, \mathrm{CaCl}_{2}\right.$, and $\left.\mathrm{MgCl}_{2}\right)$ solutions and suspensions were prepared from pure water (Elix, Millipore). The electric conductivities of the freshly purified water were around $0.5 \mu \mathrm{S} / \mathrm{cm}$.

Electrophoretic mobility (EPM) was measured by means of an improved laser Doppler velocimetry; mixed mode measurement (M3) - phase analysis light scattering (PALS) technique (Zetasizer NANO-ZS, Malvern). Details of the technique are described elsewhere [28]. Measurements of EPM were carried out as a function of salt concentration, $0.1-100 \mathrm{mM}$, and at a temperature of $298 \mathrm{~K}$. The particle concentrations were $17-34 \mathrm{mg} / \mathrm{L}$. The EPM values were insensitive to changes of particle concentration. Samples were prepared from latex suspensions by adding appropriate volumes of water and stock salt solutions to adjust the particle and salt concentrations. The values of the suspension $\mathrm{pH}$ were checked by a combination electrode (6.0234.110, Metrohm) and were $5.70 \pm 0.21$. The $\mathrm{pH}$ value was considered to be due to the dissolved carbonate from air.

\section{Modeling}

Measured electrophoretic mobilities were compared with calculated ones by using the theoretical models based on the standard electrokinetic and PoissonBoltzmann (PB) theories. Methods of modeling are written below. 
As stated above, this study used latex particles carrying a constant surface charge density $\sigma$. The surface charge density can be related to the surface potential $\psi_{0}$ through the PB equation as $[1,29]$

$$
\sigma=\frac{\varepsilon_{\mathrm{r}} \varepsilon_{0} \kappa \mathrm{k}_{\mathrm{B}} \mathrm{T}}{\mathrm{e}} \mathrm{f}\left(\mathrm{y}_{0}\right)
$$

with

$$
\begin{aligned}
& \kappa=\left(\frac{1}{\varepsilon_{\mathrm{r}} \varepsilon_{0} \mathrm{k}_{\mathrm{B}} \mathrm{T}} \sum_{\mathrm{i}=1}^{\mathrm{N}} \mathrm{z}_{\mathrm{i}}^{2} \mathrm{e}^{2} \mathrm{n}_{\mathrm{i}}\right)^{\frac{1}{2}}, \\
& \mathrm{f}(\mathrm{y})=[1-\exp (-\mathrm{y})]\left[\frac{2 \sum_{\mathrm{i}=1}^{\mathrm{N}} \mathrm{n}_{\mathrm{i}}\left[\exp \left(-\mathrm{z}_{\mathrm{i}} \mathrm{y}\right)-1\right]}{[1-\exp (-\mathrm{y})]^{2} \sum_{\mathrm{i}=1}^{\mathrm{N}} \mathrm{z}_{\mathrm{i}}^{2} \mathrm{n}_{\mathrm{i}}}\right]^{\frac{1}{2}}, \\
& \mathrm{y}_{0}=\frac{\mathrm{e} \Psi_{0}}{\mathrm{k}_{\mathrm{B}} \mathrm{T}},
\end{aligned}
$$

where $1 / \kappa$ is the Debye length in a solution containing $N$ ionic species with the valence of $i$ th ion specie $z_{i}$ and the number concentration of $i$ th ion $n_{\mathrm{i}}$. The Debye length $1 / \kappa$ is regarded as a measure of the thickness of diffuse double layer. Other parameters, $k_{\mathrm{B}}, \mathrm{T}, \varepsilon_{\mathrm{r}}, \varepsilon_{0}$, and $e$ are the Boltzmann constant, absolute temperature, the relative permittivity of liquid, the permittivity of a vacuum, and the elementary charge, respectively. When the surface potential is low, the surface charge-potential relationship, Eq. (1), can be simplified as

$$
\sigma=\varepsilon_{\mathrm{r}} \varepsilon_{0} \kappa \Psi_{0}
$$

which is given by the solution of the linearized PB equation.

Assuming that the evaluated surface potential $\psi_{0}$ equals an electrokinetic or so-called zeta potential $\zeta$, we can use the potential as an input parameter to calculate electrophoretic mobility $\mu$. With the Smoluchowski equation, one can calculate electrophoretic mobility $\mu$ in a solution with a viscosity $\eta$ using

$$
\mu=\frac{\varepsilon_{\mathrm{r}} \varepsilon_{0}}{\eta} \zeta
$$

When the potential is low, an approximated relation

$$
\mu=\frac{\sigma}{\eta \kappa}
$$


is applicable by combining Eqs. (5) and (6) [30]. Both Eqs. (6) and (7) neglect the relaxation effect retarding particle velocity. To take into account the influence of the relaxation effect, the O'Brien and White theory [13] can be used to calculate mobility of a sphere with a radius $a$ through the computer program.

Approximate analytical expressions to calculate mobility of spheres from zeta potential have been proposed [11-14]. Among them, Ohshima's expressions $[11,12]$ were adopted in this study, because they are more accurate and available in the presence of asymmetrical electrolytes. Ohshima's equations are valid when $\kappa a \geq 30$. This condition was fulfilled in the present study. Before describing Ohshima's expressions, several parameters need to be defined as follows: the drag coefficient of the $i$ th ion specie $\lambda_{\mathrm{i}}$

$$
\lambda_{\mathrm{i}}=\frac{\mathrm{N}_{\mathrm{A}} \mathrm{e}^{2}\left|\mathrm{z}_{\mathrm{i}}\right|}{\Lambda_{\mathrm{i}}^{0}},
$$

where $N_{\mathrm{A}}$ Avogadro's number and $\Lambda^{0}{ }_{\mathrm{i}}$ the limiting conductance of $i$ th ion specie, the dimensionless zeta potential

$$
\tilde{\zeta}=\frac{\mathrm{e} \zeta}{\mathrm{k}_{\mathrm{B}} \mathrm{T}},
$$

the scaled drag coefficient of ith ion specie

$$
\mathrm{m}_{\mathrm{i}}=\frac{2 \varepsilon_{\mathrm{r}} \varepsilon_{0} \mathrm{k}_{\mathrm{B}} \mathrm{T}}{3 \eta \mathrm{z}_{\mathrm{i}}^{2} \mathrm{e}^{2}} \lambda_{\mathrm{i}}
$$

Electrophoretic mobility $\mu$ in $z-z$ symmetrical electrolyte $z_{1}=-z_{2}$ solution such as $\mathrm{KCl}$ is given by

$$
\mu=\operatorname{sgn}(\zeta) \frac{\varepsilon_{\mathrm{r}} \varepsilon_{0}}{\eta}\left\{|\zeta|-\frac{2 \mathrm{~F}}{1+\mathrm{F}}\left(\frac{\mathrm{k}_{\mathrm{B}} \mathrm{T}}{\mathrm{ze}}\right) \ln \left[\frac{1+\exp (\mathrm{z}|\tilde{\zeta}| / 2)}{2}\right]\right\}
$$

with

$$
\begin{aligned}
& \mathrm{F}=\frac{2}{\kappa \mathrm{a}}(1+3 \mathrm{~m})\{\exp (\mathrm{z}|\tilde{\zeta}| / 2)-1\}, \\
& \mathrm{m}=\frac{2 \varepsilon_{\mathrm{r}} \varepsilon_{0} \mathrm{k}_{\mathrm{B}} \mathrm{T}}{3 \eta \mathrm{z}^{2} \mathrm{e}^{2}} \lambda,
\end{aligned}
$$

where $m$ is the scaled drag coefficient of counter ions and the function $\operatorname{sgn}(\mathrm{x})$ is 1 when $\mathrm{x}>0$ or -1 when $\mathrm{x}<0$.

Mobility for negatively charged particles in a 2-1 electrolyte solution like $\mathrm{CaCl}_{2}$ and $\mathrm{MgCl}_{2}$ is written by 


$$
\mu=\frac{\varepsilon_{\mathrm{r}} \varepsilon_{0}}{\eta}\left\{\zeta+\frac{2 \mathrm{~F}}{1+\mathrm{F}}\left(\frac{\mathrm{k}_{\mathrm{B}} \mathrm{T}}{\mathrm{e}}\right) \ln \left[\frac{1}{2} \exp \left(\frac{-\tilde{\zeta}}{2}\right)+\frac{1}{2} \sqrt{\frac{1}{3} \exp (-\tilde{\zeta})+\frac{2}{3}}\right]\right\}
$$

with

$$
\begin{aligned}
& \mathrm{F}=\frac{\sqrt{3}}{\kappa \mathrm{a}}\left(1+3 \mathrm{~m}_{+}\right)\{\exp (-\tilde{\zeta})-1\} \\
& \mathrm{m}_{+}=\frac{\varepsilon_{\mathrm{r}} \varepsilon_{0} \mathrm{k}_{\mathrm{B}} \mathrm{T}}{6 \eta \mathrm{e}^{2}} \lambda_{+}
\end{aligned}
$$

where $m_{+}$is the scaled drag coefficient of counter ion.

For negatively charged particles in a 1-2 electrolyte solution such as $\mathrm{Na}_{2} \mathrm{SO}_{4}$,

$$
\mu=\frac{\varepsilon_{\mathrm{r}} \varepsilon_{0}}{\eta}\left\{\zeta+\frac{2 \mathrm{~F}}{1+\mathrm{F}}\left(\frac{\mathrm{k}_{\mathrm{B}} \mathrm{T}}{\mathrm{e}}\right) \ln \left[\frac{1}{2}+\frac{1}{2} \sqrt{\frac{2}{3} \exp (-\tilde{\zeta})+\frac{1}{3}}\right]\right\}
$$

with

$$
\begin{aligned}
& \mathrm{F}=\frac{\sqrt{6}}{\kappa \mathrm{a}}\left(1+3 \mathrm{~m}_{+}\right)\{\exp (-\tilde{\zeta} / 2)-1\} \\
& \mathrm{m}_{+}=\frac{2 \varepsilon_{\mathrm{r}} \varepsilon_{0} \mathrm{k}_{\mathrm{B}} \mathrm{T}}{3 \eta \mathrm{e}^{2}} \lambda_{+}
\end{aligned}
$$

where $m_{+}$is the scaled drag coefficient of counter ion.

The Ohshima equations described above were used to evaluate electrophoretic mobilities of the latex spheres in $\mathrm{KCl}, \mathrm{Na}_{2} \mathrm{SO}_{4}, \mathrm{CaCl}_{2}$, and $\mathrm{MgCl}_{2}$ solutions. In the calculation, the values of the limiting conductance $\Lambda^{0}{ }_{\mathrm{i}}\left(10^{-}\right.$ ${ }^{4} \mathrm{Sm}^{2} \mathrm{~mol}^{-1}$ ) were 73.48, 59.47, 53.06, 76.31, and 80.0 for $\mathrm{K}^{+}$, (1/2) $\mathrm{Ca}^{2+}$, $(1 / 2) \mathrm{Mg}^{2+}, \mathrm{Cl}^{-}$, and $(1 / 2) \mathrm{SO}^{2-}$, respectively [31, 32]

\section{Results and Discussion}

The relationship between electrophoretic mobility (EPM) and $\mathrm{KCl}$ concentration is shown in Fig.1. In the figure, symbols stand for the experimental data, indicating that the magnitude of EPM shows a maximum around 1-10 mM and decreases at lower and higher $\mathrm{KCl}$ concentrations. The solid, dotted, and dashed lines were calculated by Ohshima's theory Eqs. (1) and (11), the Smoluchowski equation Eqs. (1) and (6), and the linearized Poisson-Boltzmann (PB) theory Eq. (7), respectively. Calculations were made using a constant surface 
charge density $\sigma$ of $-0.07 \mathrm{C} / \mathrm{m}^{2}$ and the assumption that the surface potential $\psi_{0}$ equals the zeta potential $\zeta$. Figure 1 demonstrates that the Smoluchowski and linearized PB theories predict the continuous decrease of the magnitude of EPM with increasing salt concentration and overestimate the absolute EPM values at lower salt concentrations. In contrast, Ohshima's theory, including the relaxation effect of electric double layer, describes the minimum in the relationship between EPM and salt concentration. In addition, Ohshima's theory quantitatively agrees well with experimental data without using any fit parameters. The reduction of absolute EPM values at lower $<1 \mathrm{mM}$ and higher $>10 \mathrm{mM}$ salt concentrations is ascribed to the relaxation effect; the relaxation must be considered to describe EPM behavior. In later figures, theoretical curves calculated with the Ohshima and PB theories are plotted.

Figure 2 demonstrates EPM behavior obtained in $\mathrm{Na}_{2} \mathrm{SO}_{4}$ solution. The symbol and line in Fig. 2 denote experimental data and theoretical calculation, respectively. While the dispersion contains divalent co-ions, the behavior is similar to those found in $\mathrm{KCl}$ solution; EPM passes through a clear minimum, and the calculated values agree with experimental data. The similarity suggests that the present physical picture of electric double layer, based on the PB equation and the relaxation of diffuse layer, is acceptable to describe EPM behavior as long as the valence of counter ions is unity.

Symbols in Fig. 3 are measured values of EPM in the presence of divalent counter-ions; $\mathrm{MgCl}_{2}$ and $\mathrm{CaCl}_{2}$. It is clear from the figures that the values of EPM taken in $\mathrm{MgCl}_{2}$ and $\mathrm{CaCl}_{2}$ are almost the same. This insignificant effect of types of divalent counter-ions is similar to the data cited by Quesada-Perez et al. [21], although Bastos and de las Nieves [20] reported that mobility of latex spheres in the presence of $\mathrm{Mg}^{2+}$ is lower than that in the presence of $\mathrm{Ca}^{2+}$. The weak dependence of EPM on kinds of divalent counter-ions implies that no specific interaction is expected between the counter-ions and the surface. The figure also indicates that the presence of divalent counter ions reduces the magnitude of EPM and makes the EPM minimum less pronounced, when compared to those obtained in the solution of monovalent counter-ions. The reason of the reduction is considered to be due to more effective screening of diffuse double layer by divalent counter-ions. Measured data are compared with theory below. 
The solid line in Fig. 3 was estimated for the EPM in $\mathrm{MgCl}_{2}$ solution by using Eqs (1) and (14) with the same assumptions as used for the monovalent counter-ion cases; a constant surface charge density $\sigma$ of $-0.07 \mathrm{C} / \mathrm{m}^{2}$ and $\psi_{0}=\zeta$. The calculated mobility values for $\mathrm{MgCl}_{2}$ are nearly identical to those for $\mathrm{CaCl}_{2}$ and thus are not shown. As seen in Fig. 3, the evaluated values agree well with experimental data at low salt concentrations 0.1-0.5 mM. At higher concentrations, however, the magnitude of calculated EPM is higher than measured ones. The disagreement suggests the need of additional effects to explain EPM behavior in the presence of divalent counter-ions. In order to decrease the discrepancy, modifications by introducing a shear plane and by reducing the magnitude of surface charge are examined.

Previous researchers have reduced the discrepancy between theory and experiment in EPM vs. concentration curves by assuming a distance $x_{\mathrm{s}}$ from the plane of the origin of surface potential $\psi_{0}$ to the plane of shear where zeta potential $\zeta$ is defined $[18,19,24-27]$. The value of $x_{\mathrm{S}}$ is regarded as the thickness of an immobile fluid layer near the surface, although how to determine the exact $x_{\mathrm{s}}$ value is still an open question. Assuming the existence of the shear plane, Borkovec et al. [18] found that calculated EPM values were in good agreement with the measured ones in $\mathrm{KCl}$ solution. Reasonable values of $x_{\mathrm{s}}$ are considered to be within the order of magnitude of sub-nanometer, which is close to the radius of hydrated ions [33]. In a solution of 2:1 electrolyte, zeta potential $\zeta$ can be evaluated from surface potential $\psi_{0}$ by the following equation:

$$
\zeta=\frac{\mathrm{k}_{\mathrm{B}} \mathrm{T}}{\mathrm{e}} \ln \left[\frac{3}{2}\left\{\frac{1+(2 / 3) \gamma \exp \left(-\kappa \mathrm{x}_{\mathrm{s}}\right)}{1-(2 / 3) \gamma \exp \left(-\kappa \mathrm{x}_{\mathrm{s}}\right)}\right\}^{2}-\frac{1}{2}\right]
$$

with

$$
\gamma=\frac{3}{2} \frac{\left[2 \exp \left(\mathrm{y}_{0}\right) / 3+1 / 3\right]^{1 / 2}-1}{\left[2 \exp \left(\mathrm{y}_{0}\right) / 3+1 / 3\right]^{1 / 2}+1} .
$$

Combining Eqs. (1), (14), and (20) with $x_{\mathrm{s}}=0.25 \mathrm{~nm}$ and $0.5 \mathrm{~nm}$ generated dotted and broken lines in Fig. 3, respectively. Figure 3 shows that the good agreement between theory and experiment is found for $x_{\mathrm{S}}=0.25 \mathrm{~nm}$. The value of $x_{\mathrm{S}}$ is comparable to radii of hydrated ions and is thus considered to be reasonable. The results validated the usefulness of the standard PB and OW (Ohshima) theories for 
the evaluation of latex EPM in the presence of divalent counter-ions. This finding contradicts the conclusion by Quesada-Perez et al [21], who suggested that the $\mathrm{PB}+\mathrm{OW}$ calculation is unable to fit mobility data even if the plane of shear is assumed, and thus that ion size correlation must be taken into account to obtain the good agreement between theoretical calculations and experimental data. The theoretical model including the effects of ion correlations also required adjusting the size of hydrated ion size to describe EPM [22]. The calculation of this work is rather simple and is therefore more practical. The existence of immobile fluid layer is probably due to the increase of counter-ions concentration in the vicinity of surface resulting from stronger attractive force between divalent counter-ions and the surface. It is plausible that raised concentration reduces the mobility of solution.

The influence of reducing the magnitude of surface charge with $x_{\mathrm{s}}=0 \mathrm{~nm}$ is shown in Fig. 4. The symbols and lines stand for experiments and calculations, respectively. The solid, dotted, and broken lines were evaluated by assuming $\sigma=-$ $0.07,-0.025$, and $-0.015 \mathrm{C} / \mathrm{m}^{2}$, respectively. It is clear from the figure that decreasing the surface charge density has an effect similar to that obtained by increasing $x_{\mathrm{s}}$ as previously reported [18]. The good agreement between theory and experiment is obtained when $\sigma=-0.025 \mathrm{C} / \mathrm{m}^{2}$. The apparent reduction of surface charge means the partial neutralization of the surface charge by adsorption of counter-ions. This also results from the stronger attractive force between divalent counter-ions and the surface.

The attraction between the divalent counter-ions and the latex surface is probably not specific but due to physical interactions, because the values of EPM in $\mathrm{MgCl}_{2}$ and $\mathrm{CaCl}_{2}$ are comparable. We need physical models to explain the existence of shear plane or reduced surface charge.

\section{Conclusion}

Electrophoretic mobilitiy (EPM) of negatively charged latex spheres bearing strong acid (sulfate) groups was measured as a function of the solution concentration and type of electrolytes. The measured values of EPM were analyzed on the basis of the standard electrokinetic model, including double layer relaxation, and the Poissonn-Boltzmann model of diffuse double layer. Calculated 
values of EPM were in good agreement with experimental data taken in simple 1:1 (KCl) and 1:2 $\left(\mathrm{Na}_{2} \mathrm{SO}_{4}\right)$ electrolyte solutions without using any fit parameters. For 2:1 $\left(\mathrm{CaCl}_{2}\right.$ and $\left.\mathrm{MgCl}_{2}\right)$ electrolytes, on the other hand, the magnitude of EPM calculated by the model overestimated the measured values of EPM in higher electrolyte concentrations. The difference between measured and calculated EPM was reduced by assuming the distance of shear plane $\mathrm{x}_{\mathrm{s}}=0.25 \mathrm{~nm}$ or by assuming the decrease of the magnitude of surface charge density from $-0.07 \mathrm{C} / \mathrm{m}^{2}$ to -0.025 $\mathrm{C} / \mathrm{m}^{2}$.

\section{Acknowledgement}

This work was financially supported by the MEXT KAKENHI (18688013). 


\section{References}

1. Ohshima H, Furusawa K ed (1998) Electrical Phenomena at Interfaces, 2nd ed. Marcel Dekker, New York

2. Masliyah JH, Bhattacharjee S (2006) Electrokinetic and Colloid Transport Phenomena, 1st ed, Wiley, Hoboken

3. Elimelech M, Gregory J, Jia X, Williams RA(1998) Particle Deposition \& Aggregation, paperback ed., Butterworth-Heinemann ,Woburn

4. von Smoluchowski M (1903) Bull Int Acad Sci Cracovie 8: 182-200

5. Henry DC (1931) Proc. Roy. Soc. London 133A: 106-129

6. Huckel E (1924) Phys. Z. 25: 204-210

7. Booth F (1950) Proc Roy Soc London 203 A: 514-533

8. Overbeek JThG (1943) Kolloide Beihefte 54:287-364

9. Wiersmema PH, Loeb AL, and Overbeek, JThG (1966) J Colloid Interface Sci 22: 78-99

10. O’Brien RW, White LR (1978) J Chem Soc, Fraday Trans 2 74: 1607-1626

11. Ohshima H, Healy TW, White LR (1983) J Chem Soc, Fraday Trans 2 79: 1613-1628

12. Ohshima H (2005) Colloids Surfaces A 267: 50-55

13. O’Brien RW, Hunter RJ (1981) Can J Chem 59: 1878-1887

14. Dukhin SS, Semenikhin NM (1970) Kolloid Zh 32: 360-368

15. Hidalgo-Alvarez R, Martin A, Fernandez A, Bastos D, Martinez F, de las Nieves (1996) Adv Coloid Interface Sci 67: 1-118

16. Elimelech M, O’Melia CR (1990) Colloids Surfaces 44: 165-178

17. Bastos-Gonzalez D, Hidalgo-Alvarez R, de las Nieves FJ (1996) J Colloid Interface Sci 177: 372-379

18. Borkovec M, Behrens SH, Semmler M (2000) Langmuir 16: 2566-2575

19. Antonietti M, Vorwerg L (1997) Colloid Polym Sci 275: 883-887

20. Bastos D, de las Nieves FJ (1993) Colloid Polym Sci 271: 860-867

21. Quesada-Perez M, Gonzarez-Tovar E., Martin-Molina A, Lozada-Cassou M. Hidalgo-Alvarez R (2005) Colloids Surfaces A 267: 24-30.

22. Martin-Molina A, Quesada-Perez M, Galisteo-Gonzalez F, Hidalgo-Alvarez R (2004) Prog Colloid Polym Sci 123: 114-118

23. Labbez C, Nonat A, Isabelle P, Jonsson B (2007) J Colloid Interface Sci 309: 303-307

24. Behrens SH, Christl DI , Emmerzael R, Schurtenberger P, Borkovec M (2000) Langmuir 16: 5209-5212

25. Lin W, Kobayashi M, Skarba M, Mu C, Galletto P, Borkovec M (2006) Langmuir 22: 1038-1047

26. Chow RS, Takamura K (1988) J Colloid Interface Sci 125: 212-225

27. Chow RS, Takamura K (1988) J Colloid Interface Sci 125: 226-236

28. Malvern Instrument (2004) Zetasizer Nano series user manual

29. Ohshima H (2006) Theory of Colloid and Interfacial Electronic Phenomena, 1st ed. Academic Press, London

30. Morisaki H, Nagai S, Ohshima H, Ikemoto E, Kogure K (1999) Microbiology 145: 2797-2802

31. Lide DR ed (2001) CRC Handbook of Chemistry and Physics, 82nd ed. CRC Press, Boca Raton

32. The Chemical Society of Japan ed (2004) Kagaku Binran, 5th ed. Maruzen, Tokyo

33. Israelachvili JN (1992) Intermolecular and Surface Forces, 2nd ed. Academic Press, London 
Figure legend

Fig. 1 Electrophoretic mobility against $\mathrm{KCl}$ concentration.

Fig. 2 Electrophoretic mobility against $\mathrm{Na}_{2} \mathrm{SO}_{4}$ concentration.

Fig. 3 Electrophoretic mobility in $\mathrm{CaCl}_{2}$ and $\mathrm{MgCl}_{2}$ concentration.

Fig. 4 Electrophoretic mobility in $\mathrm{CaCl}_{2}$ and $\mathrm{MgCl}_{2}$ concentration. 


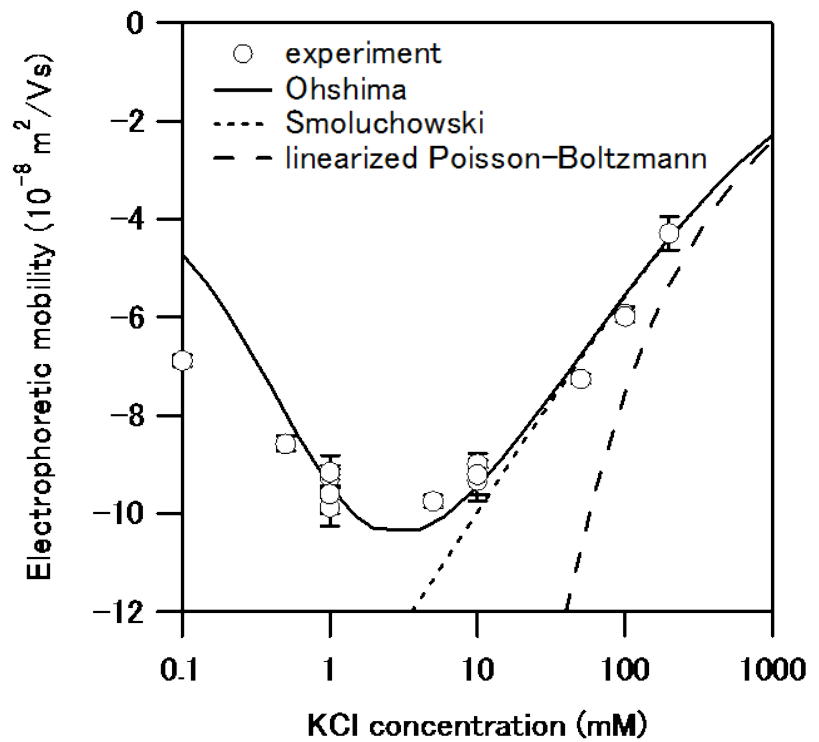

Fig.1

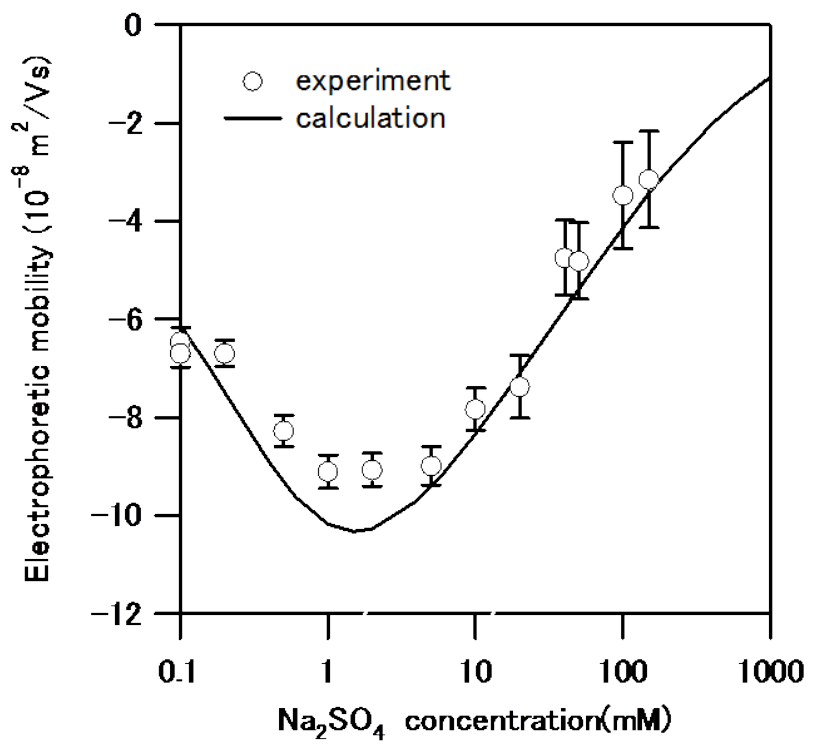

Fig. 2 


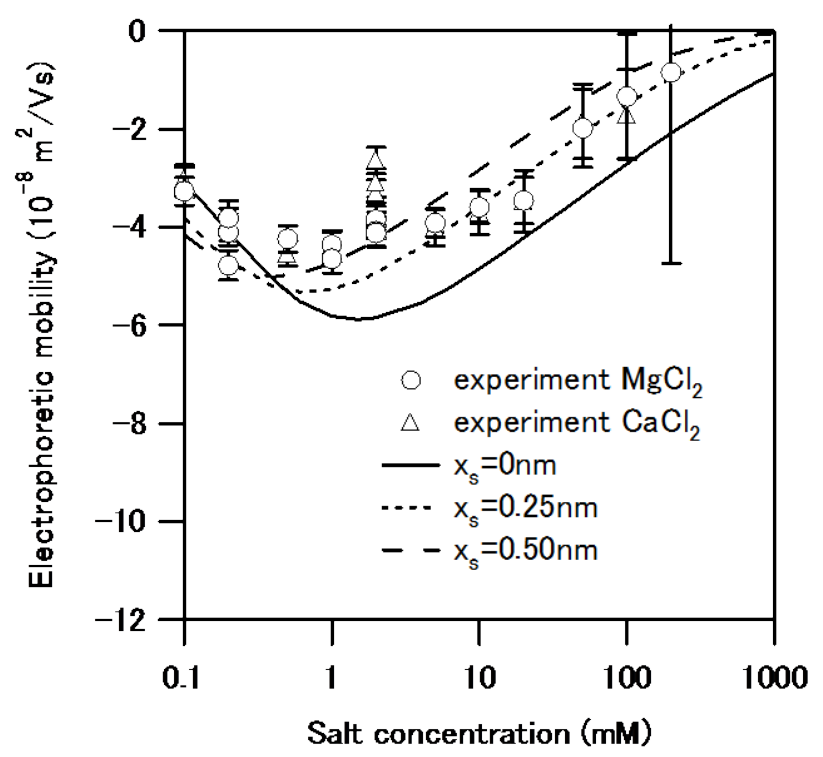

Fig. 3

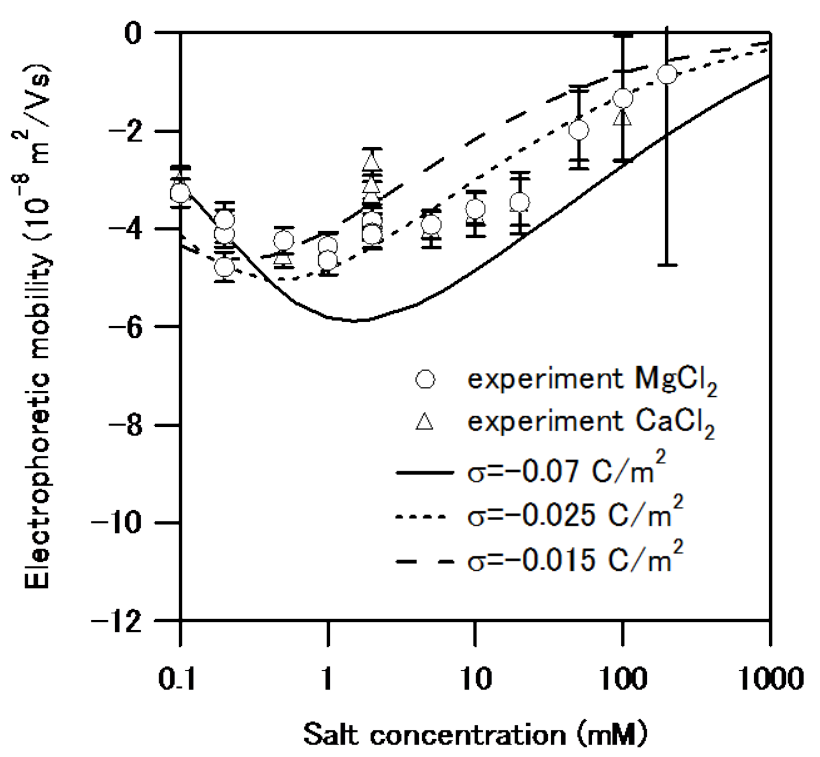

Fig. 4 